\title{
Cost-Effective Design of a Hybrid Electrical Energy Storage System for Electric Vehicles
}

\author{
Di Zhu ${ }^{1}$, Siyu Yue ${ }^{1}$, Sangyoung Park ${ }^{2}$, Yanzhi Wang ${ }^{1}$, Naehyuck Chang ${ }^{3}$, and Massoud Pedram ${ }^{1}$ \\ ${ }^{1}$ University of Southern California, USA, ${ }^{2}$ Seoul National University, ${ }^{3}$ Korea Advanced Institute of Science and Technology, Korea \\ ${ }^{1}$ \{dizhu, siyuyue, yanzhiwa, pedram\}@usc.edu, ${ }^{2}$ sypark@elpl.snu.ac.kr, 3naehyuck@cad4x.kaist.ac.kr
}

\begin{abstract}
A comprehensive economic feasibility analysis and a cost-driven design methodology are essential to the successful application of hybrid electrical energy storage (HEES) systems in electric vehicles (EVs). This paper thus focuses on designing a cost-effective and high-performance HEES system for EVs, comprising of a supercapacitor bank and a lithium-ion (Li-ion) battery bank. In particular, the paper formulates the capacity provisioning problem for the EV HEES system so as to minimize the total system cost, utilizing accurate models of the battery cycle efficiency and state of health, characteristics of the supercapacitor bank, and dynamics of the EV. The aforesaid problem is subsequently solved by combining a gradient descent-based approach with a simulated annealing-based algorithm. Simulation results show that the proposed EV HEES system achieves $21 \%$ lower total cost per day and $30 \%$ higher fuel economy compared to a baseline homogeneous electrical energy storage system comprised of Li-ion batteries only.
\end{abstract}

\section{INTRODUCTION}

Electric vehicles (EVs) have attracted significant attention of late. They outperform conventional vehicles in terms of fuel economy for the following two reasons: (i) the efficiency of an internal combustion engine (ICE) is less than 30\% [10] whereas the efficiency of an electric motor can reach $90 \%$ or even higher [13], and (ii) the kinetic energy of conventional vehicles during braking is dissipated as heat, whereas an EV is capable of recycling the braking energy to charge its electrical energy storage (EES) system, i.e., regenerative braking. The energy for braking accounts for around $30 \%$ of the total traction energy on average, and can reach up to $80 \%$ in the case of heavy city traffic [17].

As the only source of providing energy to the electric motor and recycling braking energy, an EES system is a critical part of an EV. EES systems in commercial full EVs (FEVs) commonly comprise of homogeneous battery banks, mostly lithium-ion ( $\mathrm{Li}$ ion) batteries (e.g., in Tesla cars, Nissan Leaf, Ford Focus, etc.). The performance of the EES system in an EV is therefore highly dependent on the intrinsic characteristics of Li-ion batteries. The cycle efficiency degradation due to heavy electrical loads or high charging currents, the battery aging effects, and limited battery lifetime are among the key factors that result in overall performance degradation in the EES system.

Supercapacitors, on the other hand, are able to deliver high power and achieve nearly $100 \%$ cycle efficiency [18], and exhibit negligible aging effects. Unfortunately, supercapacitors suffer from severe self-discharge (about 20\% per day [18]), low energy density, and most importantly, high capital cost, all of which pro-

This work is supported in part by a grant from the Directorate for Computer \& Information Science \& Engineering of the National Science Foundation and the Mid-Career Researcher Program and the International Research \& Development Program of the NRF of Korea funded by the MSIP (NRF-2014-023320). The SPORT lab at USC and CAD4X at KAIST provide research facilities for this study. hibit their applicability as the main energy storage element in EVs.

The idea of a hybrid EES (HEES) system, which combines Liion battery bank with a supercapacitor bank of smaller capacity as an energy buffer, has the potential to simultaneously achieve high cycle efficiency, high energy density, and acceptable system capital cost. Researchers have been exploring the optimal design and control methods of a HEES system that adopts supercapacitors as energy buffers to maximize the system performance $[2][11][15][16][20]$. However, the lack of a detailed analysis about the economic feasibility of battery/supercapacitor HEES systems in EVs has so far inhibited the successful adoption of this technology by EV manufacturers.

A convincing economic feasibility analysis should provide accurate calculation of the EV HEES system's overall cost and benefit in comparison with homogeneous EES systems for EVs. Various real-life factors affect the overall cost of an EV HEES system, including (but not limited to): (i) the capital cost of the HEES system comprising the costs of supercapacitors, batteries, and converters, (ii) the discharge/charge efficiency of the HEES system, which determines the electricity cost of the vehicle, (iii) the volume and weight of the HEES system, where the system weight also affects the electric motor power demand, and (iv) the battery capacity degradation, and therefore, the expected HEES system lifetime. The design of a cost-efficient EV HEES system must take these factors into consideration.

To the best of our knowledge, this paper is the first to address all of the aforesaid concerns about the battery/supercapacitor HEES system and present a cost-driven design and control optimization method for the HEES system in EVs. We formulate a joint optimization problem, which simultaneously optimizes the HEES system sizing and the energy flow of battery and supercapacitor banks in the HEES system so as to minimize the total cost of the EV HEES system. To be more specific, we derive the capacities of the Li-ion battery bank and the supercapacitor bank and the currents flowing from different banks of the HEES system to the electric motor under standard driving profiles from [4]. The proposed problem formulation not only takes into account the above-mentioned real-life factors but also satisfies the EV design specifications such as the all-electric range (AER) requirement. The solution is based on a combined gradient descent and simulated annealing algorithm.

The contributions of this paper are three-fold:

- Identifying key factors in the cost analysis of the HEES system with detailed EES models;

- Formulating the design and control optimization problem of a cost-efficient EV HEES system, in order to minimize the sum of the capital cost (HEES cost) and the operational cost (the cost of electricity);

- Presenting a highly effective algorithm to solve the combined design and control optimization problem.

The remaining of this paper is formulated as follows. Section 2 summarizes prior arts in EV research, and elaborates the challeng- 
Table 1. Commercialized FEVs.

\begin{tabular}{|c|c|c|c|c|c|c|c|}
\hline Model & $\begin{array}{c}\text { Battery } \\
\text { Type }\end{array}$ & $\begin{array}{c}\text { Battery } \\
\text { Capacity }\end{array}$ & EPA Range & $\begin{array}{l}\text { Battery } \\
\text { Weight }\end{array}$ & Maximum Power & $\begin{array}{l}\text { Vehicle } \\
\text { weight }\end{array}$ & Warranty \\
\hline Mitsubishi i-MiEV & Li-ion & $16 \mathrm{kWh}$ & 65 miles & - & $47 \mathrm{~kW}$ & $1.08 \mathrm{t}$ & $10 \mathrm{yrs} / 100 \mathrm{k}$ miles \\
\hline Ford Focus & Li-ion & $23 \mathrm{kWh}$ & 76 miles & $\sim 270 \mathrm{~kg}$ & $107 \mathrm{~kW}$ & $1.674 \mathrm{t}$ & $8 \mathrm{yrs} / 100 \mathrm{k}$ miles \\
\hline Nissan Leaf & Li-ion & $24 \mathrm{kWh}$ & 75 miles & - & $80 \mathrm{~kW}$ & $1.493 \mathrm{t}$ & $8 \mathrm{yrs} / 100 \mathrm{k}$ miles \\
\hline Tesla Model S & Li-ion & $60 / 85 \mathrm{kWh}$ & $208 / 265$ miles & $\sim 402 / \sim 570 \mathrm{~kg}$ & $310 \mathrm{~kW}$ & $2.108 \mathrm{t}$ & $8 \mathrm{yrs} / 125 \mathrm{k}$ miles \\
\hline
\end{tabular}

es in designing a desirable cost-effective EV HEES system. Section 3 describes the system models, and Section 4 formulates and solves the proposed problem. Simulation results are given in Section 5, and finally Section 6 concludes the paper.

\section{BACKGROUND}

\subsection{Related Work}

Recently, various types of EVs have been developed and commercialized: full EVs (FEVs), hybrid EVs (HEVs), plug-in HEV (PHEVs), and fuel cell EVs (FCEVs), etc. This paper focuses on FEV s equipped with HEES systems as the energy source for electric motors. The term "EV" in the following of this paper refers to FEV unless explicitly stated. Table 1 lists the specifications of some commercialized EV models.

Li-ion batteries have been widely accepted as the most promising energy storage technology [1][7][8]. However, they suffer from a large amount of energy dissipation during discharge/charge cycles and relatively low power output capacity [18]. The idea of hybridizing the energy storage systems in EVs to achieve higher efficiency and peak power generation has been proposed and studied for years [14][17]. Several topologies have been proposed and compared for a HEES system comprised of a NiMH bank and a supercapacitor bank for an HEV [11]. In addition, a hybrid energy storage system structure, which connects batteries and supercapacitors with the DC bus using some conversion circuitry [2], has been presented and analyzed in details along with control strategies.

The importance of analyzing the economic feasibility of the embedded HEES system has led to a number of projects emphasizing the profit maximization of HEES systems for residential usage [24][25] and for application in HEVs and PHEVs [16][12]. As for FEVs, Miller et al. propose a feedback control algorithm for a Li-ion battery/supercapacitor hybrid system, and further provide an economic analysis considering the costs of packaging, foils, and converters [15]. However, no optimization targeting at profit maximization has been proposed. Wang et al. combine the optimal design and real-time control of the HEES system in EVs [20]. However, some crucial factors such as battery aging and vehicle weight have not been considered in either the HEES sizing or the real-time control and management.

\subsection{HEES Design Challenges}

There are two major challenges in the design of a cost-effective EV HEES system. The first is the estimation of everyday operational costs of the HEES system, which is largely affected by the energy flow (i.e., the discharge/charge powers) of the banks in the HEES system and the power demand from the electric motor. To ensure the estimation accuracy, system designers should also consider battery cycle efficiency, supercapacitor self-discharge, and more importantly, battery capacity degradation (which has been ignored in most prior arts).

The second challenge is to determine the optimal sizing of the battery bank and the supercapacitor bank in the HEES system. The sizing directly affects the system's capital cost. The tricky part, which gets easily ignored, is that the daily operational costs are also influenced by the system sizing. More precisely, if we increase the battery bank size, both the cycle efficiency and battery cycle life can be improved, and the daily operational cost can be reduced but at the price of raising both the capital cost and the vehicle weight. The increased vehicle weight implies higher vehicle traction power demand and might even cancel out the operational cost reduction.

The inter-dependency between daily operational cost and system sizing makes the design problem more challenging. On one hand, the bank capacities should be adjusted based on the optimization result of energy flow control. On the other hand, the EV traction power demand also changes as the sizes of the battery bank and the supercapacitor bank are getting adjusted, and therefore, the optimal energy flows have to be re-calculated to maximize the cycle efficiency. Such circular dependency makes the design problem more difficult.

In order to solve the above problem, we consider the overall HEES design and control problem in a unified way to simultaneously optimize the bank sizing and minimize daily operational costs.

\section{SYSTEM MODEL}

Figure 1 shows the overview of the proposed EV HEES system. It consists of an electric traction motor, a HEES system and power conversion circuitry. Note that the power flow between the HEES system and the motor is bidirectional: the HEES system provides power to the motor when the vehicle is accelerating or cruising, and collects power from the motor when the vehicle is braking. The details of the vehicle dynamics model and HEES system are given in the following subsections.

\subsection{Vehicle Dynamics Model}

This paper adopts the well-known vehicle dynamics model [21]. It computes the total traction force $F_{T}$ of a vehicle by

$$
F_{T}=F_{a}+F_{g}+F_{R}+F_{A D}
$$

$=m \cdot a+m \cdot g \cdot \sin \theta+C_{r r} \cdot m \cdot g \cdot \cos \theta+\frac{1}{2} \cdot C_{A} \cdot \rho \cdot A \cdot v^{2}$,

where $F_{a}$ denotes the acceleration force, $F_{g}$ is the vertical component of vehicle gravity force on a road with slope $\theta, F_{R}$ is rolling friction force, $F_{A D}$ denotes the aerodynamic resistance. $m$ denotes the vehicle total mass, and $a$ the vehicle acceleration, $\theta$ the gradi-

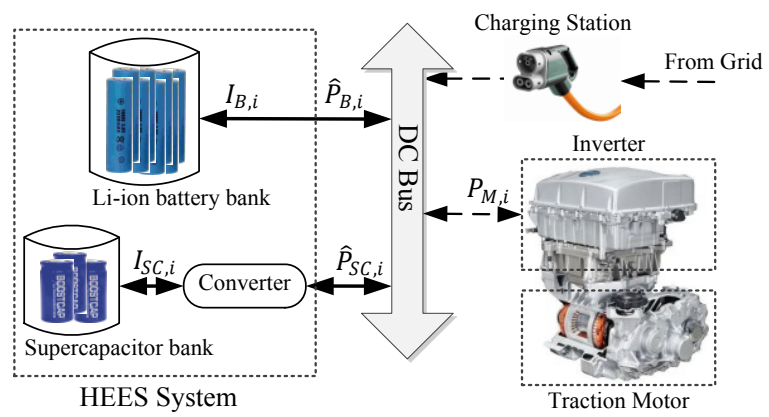

Figure 1. HEES architecture. 
Table 2. Symbols used in the problem formulation.

\begin{tabular}{|c|l|}
\hline$M_{0}$ & Vehicle base mass without the HEES system \\
\hline$f_{c o n v}$ & Converter efficiency as a function of $V_{\text {out }}, I_{i n}, V_{i n}$ \\
\hline$f_{O C V}$ & OCV of Li-ion battery as a function of SoC \\
\hline$Q_{B}\left(Q_{S C}\right)$ & Battery (or SC) FCC \\
\hline$I_{B, i}\left(I_{S C, i}\right)$ & Battery (or SC) output current in the $i$-th time slot \\
\hline$I_{B, i}^{i n t}$ & Battery internal charge loss rate in the $i$-th time slot \\
\hline$V_{B, i}^{O C}\left(V_{S C, i}^{O C}\right)$ & Battery (or SC) OCV at the $i$-th decision epoch \\
\hline$V_{B, i}^{C C}\left(V_{S C, i}^{C C}\right)$ & Battery (or SC) CCV at the $i$-th decision epoch \\
\hline$k$ & Peukert's constant \\
\hline$R_{B}^{i n t}\left(R_{S C}^{i n t}\right)$ & Internal resistance of battery (or SC) \\
\hline$\rho_{B}^{E}\left(\rho_{S C}^{E}\right)$ & Specific energy density (in Wh/kg) of battery (or SC) \\
\hline$\rho_{B}^{V}\left(\rho_{S C}^{V}\right)$ & Volumetric energy density (Wh/L) of battery (or SC) \\
\hline$\rho_{B}^{P}\left(\rho_{S C}^{P}\right)$ & Specific power density (in $\mathrm{W} / \mathrm{kg})$ of battery (or SC) \\
\hline$V_{B, \text { nom }}^{O C}$ & Nominal OCV of the battery bank \\
\hline$S_{B}$ & $\begin{array}{l}\text { The initial SoC swing, i.e., SoC change in a dis- } \\
\text { charge/charge cycle of a fresh battery }\end{array}$ \\
\hline$\eta_{S C}$ & Efficiency of the supercapacitor bank converter \\
\hline$C_{S C}$ & Capacitance of the supercapacitor bank \\
\hline$V_{S C, m a x}^{O C}$ & Supercapacitor maximum output voltage \\
\hline
\end{tabular}

ent angle, $C_{r r}$ the rolling resistance coefficient, $C_{A}$ the aerodynamic resistance coefficient, $\rho$ the air density, $A$ the vehicle frontal area, $v$ the vehicle velocity.

Given the total traction force, we calculate the driving power demand by

$$
P_{d}=F_{T} \cdot v / \eta_{E M}
$$

$\eta_{E M}$ denotes the overall efficiency of electric motor (the inverter included). It is a function of the motor angular speed $\omega_{E M}$ (calculated by $\left.\omega_{E M}=v \cdot \mu / r\right)$ and the motor torque $T_{E M}=F_{T} \cdot r / \mu$, where $r$ is the tire radius and $\mu$ the axle ratio.

The HEES system sizing has an influence on the total mass $m$ of the vehicle, therefore influencing the driving power demand.

\subsection{Energy Storage Characteristics}

Table 2 lists the symbols used hereinafter. For EES bankrelated variables, we use $B$ in their subscripts to stand for the battery bank and $S C$ for the supercapacitor bank. For example, $I_{B}$ is the battery bank output current and $I_{S C}$ is the supercapacitor bank output current.

We first summarize the terms of energy storage characteristics as follows.

Full charge capacity (FCC) of a battery is defined as the rated capacity when it is fully charged. Due to capacity degradation, a cycle-aged battery's FCC is less than its designed capacity (also called the nominal FCC).

A discharge/charge cycle (or $d / c$ cycle) refers to one time of discharging an EES element and then charging it. A driving cycle refers to the period of time during which an EV executes a complete driving profile (which is, in our simulation, a combination of several consecutive standard vehicle profiles defined in [4]).

State-of-charge (SoC) of a battery is the ratio of the remaining amount of stored charge to the current FCC (instead of the nominal FCC). The SoC swing refers to the SoC change during a dis- charge/charge cycle. The open circuit voltage (OCV) of a battery is a nonlinear increasing function $f_{O C V}$ of the SoC [19].

\subsubsection{Cycle Efficiency}

Li-ion batteries suffer cycle efficiency degradation due to rate capacity effect. Peukert's law shows that the internal charge loss (or increase) in a battery is a superlinear (or sublinear) function of its output discharge (or charge) current $I_{B}[9]$, i.e.,

$$
I_{B}^{i n t}=\left(\frac{I_{B}}{I_{\text {ref }}}\right)^{k} I_{\text {ref }}
$$

where $k>1$ during battery discharging and $k<1$ during battery charging, and $I_{\text {ref }}=Q_{B} / 20$ for a battery with FCC of $Q_{B}$ (in Ah).

\subsubsection{Self-Discharge}

Supercapacitors suffer severe self-discharge effect. A supercapacitor loses over $20 \%$ of its stored energy per day even without any load [18]. The time scale in EV application is typically in hours, and therefore, the self-discharge effect is taken into consideration. The OCV decay of a supercapacitor after $\Delta t$ time with no load is calculated by

$$
V_{S C}^{O C}(t+\Delta t)=V_{S C}^{O C}(t) \cdot e^{-\Delta t / \tau}
$$

where $\tau$ is the self-discharge time constant.

\subsubsection{Internal Resistance Loss (IR Loss)}

Both batteries and supercapacitors have internal resistance. We calculate the closed-circuit voltage by

$$
V_{B}^{C C}=V_{B}^{O C}-I_{B} R_{B}^{i n t}, V_{S C}^{C C}=V_{S C}^{O C}-I_{S C} R_{S C}^{i n t} .
$$

The internal resistance of a Li-ion battery is a function of the battery's SoC, but is almost constant for SoC greater than 0.1 [3]. Li-ion batteries also experience the exacerbation of internal resistance $R_{B}^{i n t}$ with aging. $R_{B}^{i n t}$ increases by typically $30 \%$ to $80 \%$ during the lifetime [5][23]. For simplicity, we use the average value of $R_{B}^{\text {int }}$ over a battery's lifetime.

\subsubsection{Energy/Power Density}

The energy (or power) density of an EES element is defined as the amount of stored energy (or rated output power) per unit mass or volume. There are three sets of density-related parameters that are crucial in the design of the HEES system for an EV, namely specific energy density, volumetric energy density, and specific power density.

Specific energy densities $\rho_{B}^{E}$ and $\rho_{S C}^{E}$ (in $\mathrm{Wh} / \mathrm{kg}$ ) directly affect the traction power demand of the EV because the overall mass of the HEES bank is calculated by $Q_{B} V_{B, \text { nom }}^{O C} / \rho_{B}^{E}+\frac{1}{2} Q_{S C} V_{S C, \text { nom }}^{O C} /$ $\rho_{S C}^{E}$. As mentioned in Section 3.1, the power demand of the electric motor $P_{M}$ is a function of the overall vehicle mass. Volumetric energy density $\rho_{B}^{V}$ and $\rho_{S C}^{V}$ (in $\mathrm{Wh} / \mathrm{L}$ ) are used for calculation of the HEES volume, which has an upper bound because of the limited space in an EV. Specific power densities $\rho_{B}^{P}$ and $\rho_{S C}^{P}$ (in $\mathrm{W} / \mathrm{kg}$ ) are used in judging whether the $\mathrm{EV}$ is able to provide the required maximum power output. As shown in Table 3, supercapacitors have around 50 times higher power density than Li-ion batteries, but only about one tenth the energy density.

Table 3. Energy/Power Density.

\begin{tabular}{|c|c|c|c|}
\hline Density & $\boldsymbol{\rho}^{\boldsymbol{E}}(\mathbf{W h} / \mathbf{k g})$ & $\boldsymbol{\rho}^{\boldsymbol{V}}(\mathbf{W h} / \mathbf{L})$ & $\boldsymbol{\rho}^{\boldsymbol{P}}(\mathbf{W} / \mathbf{k g})$ \\
\hline Li-ion battery & $100-250$ & $250-620$ & $250-1500$ \\
\hline Supercapacitor & $2.5-15$ & $5-15$ & $15 \mathrm{k}-99 \mathrm{k}$ \\
\hline
\end{tabular}

\subsubsection{Cycle Life}

The cycle life of an EES element is defined as the number of $\mathrm{d} / \mathrm{c}$ cycles it can undergo before its FCC degrades below a certain 


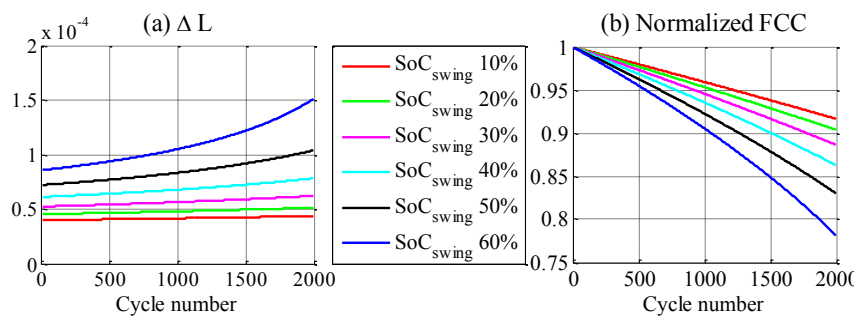

Figure 2. Li-ion battery capacity degradation with the same amount of charge loss in each $\mathrm{d} / \mathrm{c}$ cycle.

threshold. The cycle life of a Li-ion battery is a function of the average SoC and SoC swing. A larger SoC swing and/or higher average $\mathrm{SoC}$ generally lead to a shorter cycle life [22].

Assume the same amount of charge is extracted from a Li-ion battery during each $\mathrm{d} / \mathrm{c}$ cycle. Let $\Delta L$ denote the percentage of the amount of FCC loss in one cycle and $Q_{B}$ denote the nominal FCC. Figure 2 shows $\Delta L$ and the FCC (normalized to $Q_{B}$ ) as a function of the cycle count with different initial SoC swing $s_{B}$ values. Note that the actual SoC swing in each cycle increases as the battery ages because the battery's FCC gradually decreases while the amount of charge extracted in each cycle remains unchanged.

One crucial observation from Figure 2 is that the change in $\Delta L$ as the cycle number increases is no more than $30 \%$ for $s_{B}$ less than 0.5 . For an EV with a range of more than 100 miles, everyday commuting hardly uses more than half of the full range battery capacity. Therefore with little loss of accuracy, we assume that Li-ion batteries experience the same amount of capacity degradation $\Delta L\left(s_{B}\right)$ in each $\mathrm{d} / \mathrm{c}$ cycle, i.e., $\Delta L\left(s_{B}\right)$ is fixed and only depends on $s_{B}$.

Supercapacitors, on the other hand, experience negligible capacity degradation, and have a cycle life long enough to operate throughout the entire vehicle lifetime.

\subsubsection{HEES Architecture}

The battery bank and the supercapacitor bank in a HEES system are connected either in parallel or in series [11]. We adopt a parallel configuration as shown in (Figure 1) with Li-ion battery bank connected to the DC bus directly. This architecture provides fine control on the terminal voltage of the supercapacitor bank as well as high power efficiency [11]. Note that the efficiency of the bi-directional power converter between the supercapacitor bank and DC bus $\eta_{S C}$ is a function of the input and output current and voltage [17], specifically

$$
\eta_{S C}=f_{\text {conv }}\left(V_{B}^{C C}, I_{S C}, V_{S C}^{C C}\right) .
$$

\section{PROBLEM STATEMENT}

The objective of this paper is to determine the optimal design of the HEES system to achieve the highest economic efficiency, i.e., determine capacities of the two banks $Q_{B}$ and $Q_{S C}$ (both in Ah). Note that the capacity of the supercapacitor bank $Q_{S C}$ is defined as $C_{S C} \cdot V_{S C, \text { max }}^{O C}$ in this paper.

To derive the optimal HEES system design, we need to estimate the system cost. More precisely, the amortized system cost comprises of two components: the electricity cost to charge the battery bank for a d/c cycle and the capital cost of the HEES system amortized to a d/c cycle.

One must obtain complete driving profiles of the EV in each day during its entire lifetime to accurately calculate the total cost, an undertaking which is clearly impossible. Thus, to model the driving pattern of a regular commuter, we make the assumptions that (i) the EV undergoes two standard driving cycles every day and (ii) it is fully charged at the end of the day. Therefore, each day the EV undergoes exactly one $\mathrm{d} / \mathrm{c}$ cycle, which contains two driving cycles.

We then formulate the EV HEES system design problem as an optimization problem to minimize the amortized system cost subject to appropriate constraints (see below).

\subsection{Problem Formulation}

We divide the entire time horizon of the standard driving profile into $N$ time slots, each of length $\Delta t$. Decision epochs are at the beginning of each time slot, when the HEES system controller decides the charge and discharge currents of storage banks in the subsequent time slot.

\section{Variables:}

1. Battery bank capacity $Q_{B}$ and supercapacitor capacity $Q_{S C}$;

2. Charge and discharge currents of battery and supercapacitor bank in each time slot: $I_{B, i}, I_{S C, i}, i \in[1, N]$. (A positive current means the bank is discharging, whereas a negative value means charging.)

Objective function: This is the $\mathrm{d} / \mathrm{c}$ cycle-amortized total system cost, denoted by $C_{\text {tot }}$, which is the sum of (i) the electricity cost per $\mathrm{d} / \mathrm{c}$ cycle to charge the batteries, $C_{E}$ and (ii) the capital cost amortized to each $\mathrm{d} / \mathrm{c}$ cycle, $C_{C}$ :

$$
C_{\text {tot }}=C_{E}+C_{C} \text {. }
$$

\subsubsection{Electricity Cost Calculation}

The electricity $\operatorname{cost} C_{E}$ of one d/c cycle is proportional to the total energy consumption $E_{c s p}$ during driving:

$$
\begin{gathered}
C_{E}=\frac{1 \mathrm{kWh}}{3600000 \mathrm{~J}} \cdot \frac{p_{E}}{\eta_{G}} \cdot E_{c s p}, \\
E_{c s p}=\sum_{i=1}^{N}\left(V_{B, i}^{O C} \cdot I_{B, i}^{i n t} \cdot \Delta t\right)+\frac{1}{2} C_{S C}\left(\left(V_{S C, 0}^{O C}\right)^{2}-\left(V_{S C, N}^{O C}\right)^{2}\right) .
\end{gathered}
$$

For simplicity we assume constant grid-charging efficiency $\eta_{G}$, and constant electricity unit price $p_{E}$ (in $\$ / \mathrm{kWh}$ ) during charging. $I_{B, i}^{\text {int }}$ is calculated according to Peukert's law (3). As mentioned in Section 3.2 , the battery OCV $V_{B, i}^{O C}$ is a function of the battery SoC at the $i$-th decision epoch, i.e.,

$$
V_{B, i}^{O C}=f_{O C V}\left(1-\frac{\sum_{j=1}^{i}\left(I_{B, j}^{i n t} \cdot \Delta t\right)}{Q_{B}}\right) .
$$

$V_{S C, i}^{O C}$ is calculated by (considering self-discharge)

$$
V_{S C, i}^{O C}=V_{S C, i-1}^{O C} \cdot e^{-\frac{\Delta t}{\tau}}-\frac{I_{S C, i} \cdot \Delta t}{C_{S C}},
$$

where $V_{S C, 0}^{O C}=V_{S C, \text { max }}^{O C}$ implies that the supercapacitor is fully charged at the beginning of the $\mathrm{d} / \mathrm{c}$ cycle. $V_{S C, N}^{O C}$ is therefore calculated recursively based on (11).

\subsubsection{Amortized Capital Cost Calculation}

The HEES capital cost is the major component of the total system cost of the EV energy storage system. Therefore, we must take it into consideration to provide a fair comparison of the overall cost of EVs against ICE-based vehicles.

The capital cost of the HEES system is comprised of four parts: the cost of the batteries $p_{B} Q_{B}$, the cost of supercapacitors $p_{S C} Q_{S C}$, the cost of other miscellaneous HEES components $C_{m i s c}$, and the 
maintenance cost $C_{M}$. Here $p_{B}$ and $p_{S C}$ denote the unit prices (in $\$ / \mathrm{Ah}$ ) of batteries and supercapacitors, respectively.

As mentioned above, the capital cost of a storage bank along with the corresponding maintenance cost should be amortized over their lifetime. Time value of money should be considered in the amortization because the lifetime of an EV is expected to be more than ten years. We introduce a depreciation factor $\gamma(\gamma<1)$. More specifically, if amortizing an amount of initial cost $A$ over $L$ $\mathrm{d} / \mathrm{c}$ cycles, the cost $C$ per cycle is calculated by

$$
\begin{gathered}
C+C \cdot \gamma+\cdots+C \cdot \gamma^{L-1}=A, \\
C=A \cdot \frac{1-\gamma}{1-\gamma^{L}} .
\end{gathered}
$$

One important observation is that the Li-ion battery has much shorter lifetime (typically 5 to 10 years) than a designed EV lifetime $L_{E V}$ (typically 10 to 20 years). Therefore, we should amortize the battery $\operatorname{cost} p_{B} Q_{B}$ (as well as the maintenance $\operatorname{cost} C_{M}$ from replacing the battery bank) over the battery lifetime $L_{B}$, while the supercapacitor cost $p_{S C} Q_{S C}$ and the cost of other miscellaneous HEES components $C_{m i s c}$ should be amortized over the EV lifetime $L_{E V}$ (in the number of $\mathrm{d} / \mathrm{c}$ cycles), i.e.,

$$
C_{C}=\left(p_{B} Q_{B}+C_{M}\right) \cdot \frac{1-\gamma}{1-\gamma^{L_{B}}}+\left(C_{m i s c}+p_{S C} Q_{S C}\right) \cdot \frac{1-\gamma}{1-\gamma^{L_{E V}}} .
$$

The battery cycle life $L_{B}$ is defined as the number of cycles the battery bank undergoes when the battery bank becomes unable to satisfy the AER requirement of the EV due to battery capacity degradation. In other words, the maximum amount of energy stored in the degraded batteries can no longer provide enough energy to drive the EV for the distance specified by AER. An assumption we made in computing $L_{B}$ is that for given EV and HEES system specifications, the charge/discharge efficiency $\eta_{H}$ of the HEES system is constant. The overall HEES system efficiency $\eta_{H}$ in one driving profile is the ratio of the energy $E_{M}$ delivered to the motor to the total energy consumption $E_{c s p}$ in the HEES (given by (9)), i.e.,

$$
\eta_{H}=\frac{E_{M}}{E_{c s p}}=\frac{\sum_{i=1}^{N} P_{M, i} \Delta t}{E_{c s p}} .
$$

$P_{M, i}$ is the electric motor power demand in the $i$-th time slot. Note that $P_{M, i}$ is a function of $Q_{B}, Q_{S C}$, and vehicle speed.

According to the aforesaid definition of the battery cycle life $L_{B}$, we have

$$
Q_{B} \cdot\left(1-L_{B} \cdot \Delta L\left(s_{B}\right)\right) \cdot V_{B, \text { nom }}^{O C}=\frac{E_{M}^{A E R}}{\eta_{H}},
$$

where $V_{B, \text { nom }}^{O C}$ is the nominal terminal voltage of the battery bank and $E_{M}^{A E R}$ denotes the motor energy demand of the AER constraint test profile [6]. Note that $E_{M}^{A E R}$ is usually much larger than $E_{M}$ (the energy demand of one $\mathrm{d} / \mathrm{c}$ cycle) and also depends on the sizes of both storage banks because of the vehicle weight. The SoC swing $s_{B}$ in a $\mathrm{d} / \mathrm{c}$ cycle is the ratio of the sum of charge loss due to discharging currents to the nominal FCC $Q_{B}$, i.e.,

$$
s_{B}=\frac{1}{Q_{B}} \sum_{I_{B, j}^{i n t}>0} I_{B, j}^{i n t} \Delta t .
$$

As mentioned in Section 3.2.5, the Li-ion battery capacity is assumed to experience linear fading effect, where the capacity degradation in every $\mathrm{d} / \mathrm{c}$ cycle is given by $\Delta L\left(s_{B}\right)$, and finally the FCC after $L_{B}$ cycles is calculated by $Q_{B} \cdot\left(1-L_{B} \cdot \Delta L\left(s_{B}\right)\right)$.

\subsubsection{Optimization Constraints}

The optimization variables must satisfy the following global and transient constraints.

\section{Global Constraints:}

1. System volume constraint, which denotes that the overall HEES volume should not exceed the designed space limit $S V$, otherwise the HEES system cannot fit into a given vehicle:

$$
Q_{B} V_{B, \text { nom }}^{O C} / \rho_{B}^{V}+\frac{1}{2} Q_{S C} V_{S C, \text { max }}^{O C} / \rho_{S C}^{V} \leq S V .
$$

2. Maximum power output requirement, which means the HEES system must be able to provide the power of a designed value $M P$ to the motor:

$$
Q_{B} V_{B, \text { nom }}^{O C} \rho_{B}^{P} / \rho_{B}^{E}+\frac{1}{2} Q_{S C} V_{S C, \max }^{O C} \rho_{S C}^{P} / \rho_{S C}^{E} \geq M P
$$

Transient Constraints: In the $i$-th time slot $(\forall i \in[1, N])$, the optimization variables must satisfy the following constraints.

3. Power delivery constraints:

$$
\hat{P}_{B, i}+\hat{P}_{S C, i}=P_{M, i},
$$

where $\hat{P}_{B, i}$ and $\hat{P}_{S C, i}$ are the power delivered to the DC bus (as shown in Figure 1) from the battery bank and the supercapacitor bank, respectively, calculated by

$$
\begin{gathered}
\hat{P}_{B, i}=V_{B, i}^{C C} \cdot I_{B, i} \\
\hat{P}_{S C, i}=\left\{\begin{array}{l}
V_{S C, i}^{C C} \cdot I_{S C, i} \cdot \eta_{S C}, \text { if } I_{S C, i} \geq 0 \\
V_{S C, i}^{C C} \cdot I_{S C, i} / \eta_{S C}, \text { if } I_{S C, i}<0
\end{array}\right.
\end{gathered} .
$$

The load power $P_{M, i}$ is a function of the sizing variables $Q_{B}, Q_{S C}$ as mentioned Section 3.2.4. Note that $P_{M, i}$ is negative when regenerative braking is applied.

4. Battery capacity constraints, i.e., the stored charge in the battery bank should neither exceed its capacity, nor fall below the minimum protective $\mathrm{SoC}$ level $S_{o} C_{B, \min }$ :

$$
\operatorname{SoC}_{B, \min } \cdot Q_{B} \leq Q_{B}-\sum_{j=1}^{i} I_{B, j}^{i n t} \Delta t \leq Q_{B} \text {. }
$$

5. Supercapacitor capacity constraints, i.e., the stored charge in the supercapacitor bank should neither exceed its capacity $Q_{S C}=C_{S C} V_{S C, \text { max }}^{O C}$ nor below $C_{S C} V_{S C, \text { min }}^{O C}$ :

$$
V_{S C, \min }^{O C} \leq V_{S C, i}^{O C} \leq V_{S C, \text { max }}^{O C}
$$

\subsection{Solution Method}

Because of the high complexity and the large number of variables (if we use $\Delta t=1 \mathrm{~s}$, a typical driving profile US06 of 8 miles [4] contains several hundreds of decision variables), we adopt a combined gradient descent and simulated annealing method to solve the above problem. First, we define the kernel problem as the above optimization problem but with given storage bank capacities $Q_{B}, Q_{S C}$. With the kernel problem solved, the optimal capacities $Q_{B}, Q_{S C}$ are then determined by the gradient descent method.

To solve the kernel problem, we divide the driving profile into multiple discharge intervals (consecutive periods of positive power demand, i.e., $P_{M, i} \geq 0$ ) and charge intervals (consecutive periods of negative power demand, i.e., $\left.P_{M, i}<0\right)$. Assuming the $\operatorname{OCV}\left\{V_{S C, j}^{O C}\right\}$ of the supercapacitor bank at the end of each interval has been determined, we apply the following heuristic algorithm to calculate the currents of both the battery bank and the supercapacitor bank.

The energy provided by/to the supercapacitor bank during the $j$ th discharge/charge interval is derived from the energy difference at the beginning and end of the interval, which can be directly calculated with given $V_{S C, j}^{O C}$ and $V_{S C, j-1}^{O C}$. As mentioned in Section 3.2.1, the discharge/charge efficiency decreases as the absolute 


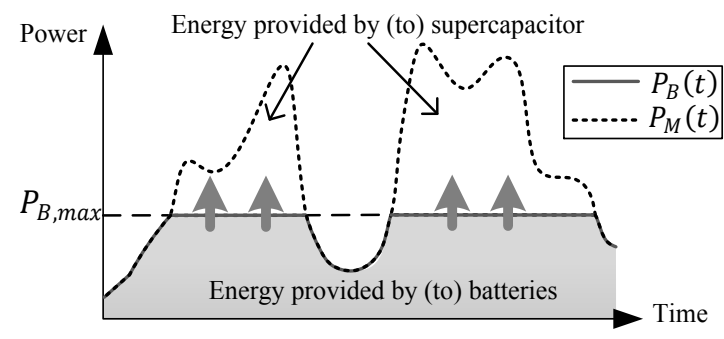

Figure 3. Water-filling strategy in one discharge (charge) interval.

value of current increases. Therefore, we keep the battery current as low as possible by deploying a water-filling strategy, described as follows.

In discharging intervals, both banks get discharged. We limit the maximum battery bank output power by $P_{B, \max }$, as shown in Figure 3 . The dotted curve denotes the electric motor power demand, and the shadowed area denotes the energy provided by/to the battery bank. We gradually increase the water level (i.e., $\left.P_{B, \max }\right)$ until the energy provided by the supercapacitor bank matches the given start and end supercapacitor OCVs of the $j$-th interval, i.e., $V_{S C, j-1}^{O C}$ and $V_{S C, j}^{O C}$.

The charging interval management gives supercapacitors the priority to get charged from the regenerative braking. If the energy from regenerative braking is sufficient to meet the supercapacitor energy demand, the battery bank gets charged, too. In this case, we employ the water-filling strategy which gradually increases the maximum battery charging power. Meanwhile, the battery is also allowed to charge supercapacitors in the charging intervals (to prepare for future power peaks). We discharge the battery bank with a constant current to provide the required energy to supercapacitors in this case.

The heuristic algorithm makes the optimization problem focus on the determination of the OCVs of the supercapacitor bank at the end of each interval in addition to the capacity of both storage banks. This allows us to reduce the number of optimization variables of the kernel problem from several hundreds to a few dozens.

Simulated annealing, which is a search-based algorithm, is employed to find the near-optimal solution of the variables $\left\{V_{S C, j}^{O C}\right\}$. Each move of simulated annealing involves adjusting one of the

Table 4. Four EV Design Requirements.

\begin{tabular}{|l|c|c|c|c|}
\hline \multicolumn{1}{|c|}{ Model } & V1 & V2 & V3 & V4 \\
\hline Range (miles) & 100 & 100 & 100 & 200 \\
\hline System Volume Constraint (L) & 150 & 200 & 250 & 350 \\
\hline EES Maximum Power (kW) & 100 & 200 & 300 & 300 \\
\hline
\end{tabular}

Table 5. EV and HEES Related Parameters.

\begin{tabular}{|l|l|l|l|}
\hline Vehicle base mass $(\mathrm{kg})$ & 1350 & Li-ion battery $p_{B}(\$ / \mathrm{kWh})$ & 200 \\
\hline
\end{tabular}

\begin{tabular}{|l|l|l|l|}
\hline Miles per driving cycle & 33.0 & Supercapacitor $p_{S C}(\$ / \mathrm{kWh})$ & 3000 \\
\hline
\end{tabular}

\begin{tabular}{|l|l|l|l|}
\hline EV lifetime (years) & 15 & Converter cost $C_{\text {conv }}(\$)$ & 200 \\
\hline Electicity
\end{tabular}

\begin{tabular}{|l|l|l|l|}
\hline Electricity cost $(\$ / \mathrm{kWh})$ & 0.12 & Battery OCV $V_{B, \text { nom }}^{O C}(\mathrm{~V})$ & 370 \\
\hline
\end{tabular}

\begin{tabular}{|l|l|l|l|}
\hline Gas cost (\$/gallon) & 3.3 & Supercap OCV $V_{S C, \text { max }}^{O C}(\mathrm{~V})$ & 400 \\
\hline
\end{tabular}

Table 6. Vehicle Dynamics Parameters.

\begin{tabular}{|l|l|l|l|}
\hline $\begin{array}{l}\text { Rolling resistance coef- } \\
\text { ficient } C_{r r}\end{array}$ & 0.01 & $\begin{array}{l}\text { Aerodynamic resistance } \\
\text { coefficient } C_{A}\end{array}$ & 0.28 \\
\hline Air density $\rho\left(\mathrm{kg} / \mathrm{m}^{3}\right)$ & 1.23 & Vehicle frontal area $A\left(\mathrm{~m}^{2}\right)$ & 0.65 \\
\hline Tire radius $r(\mathrm{~m})$ & 0.35 & Axle ratio $\mu$ & 7.94 \\
\hline
\end{tabular}

supercapacitor OCVs $\left\{V_{S C, j}^{O C}\right\}$. If the move is accepted, the charge or discharge currents in the two adjacent intervals are updated according to the above-mentioned water-filling strategy.

\section{SIMULATION RESULTS}

\subsection{Simulation Setup}

The simulation evaluates the proposed EV HEES system design and control methodology with four different EV design requirements summarized in Table 4. These design requirements are similar to those listed in Table 1 to make the simulation more realistic. The range of an EV is determined as stated in the ETA standard [6]. To be specific, in a range test, the EV is fully charged, parked overnight, and in the following day driven over successive standard driving profiles until the EES system becomes depleted and the EV can no longer follow the driving cycle.

We adopt an EV motor efficiency map from ADVISOR [13]. Other specifications of the EV HEES system are listed in Table 5, and the vehicle dynamics that are common for the four EV models $\mathrm{V} 1$ to V4 are listed in Table 6. Each driving cycle contains four dynamometer driving schedules, namely FTP, HWY, US06 and $\mathrm{SC0} 3$ [4], to form a combination of city and highway, aggressive and conservative driving profiles. The total length of one driving cycle is 33 miles.

In addition to the total cost per $\mathrm{d} / \mathrm{c}$ cycle defined in Section 4 , we also compare the fuel economy in the simulation results. Fuel economy implies the fuel efficiency of a vehicle. The Environmental Protection Agency (EPA) uses miles per gallon (MPG) to denote the fuel economy of an ICE-powered vehicle, while the fuel economy of an EV is quantified by miles per gallon gasoline equivalent (MPGe). The conversion standard in calculating MPGe is that one gallon of gasoline equals $33.7 \mathrm{kWh}$ of electricity.

\subsection{Comparison with Homogenous EES}

Table 7 elaborates the optimization results of the homogenous EES systems and the proposed HEES systems for the four different EV specifications. Figure 4(a) and (b) visualize the total cost per day (i.e., per d/c cycle) and MPGe results (both normalized to the cost or MPG of ICE vehicles), and (c) and (d) show the system volume and mass results. The designed HEES system saves the amortized cost per day by averagely $20.91 \%$, increases the fuel economy by $30.29 \%$, reduces system mass by $16.98 \%$, and shrinks system volume by $9.3 \%$ on average compared to the homogeneous EES system. We have the following conclusions from the results:

1) The capacities of EV HEES system are largely influenced by

Table 7. Simulation Results.

\begin{tabular}{|c|c|c|c|c|c|}
\hline \multicolumn{2}{|r|}{ Model } & V1 & V2 & V3 & V4 \\
\hline \multirow{5}{*}{$\begin{array}{l}\text { Homo- } \\
\text { geneous }\end{array}$} & $Q_{B}(\mathrm{Ah})$ & 114 & 120.1 & 180.2 & 228 \\
\hline & EES volume (L) & 141 & 148 & 222 & 281 \\
\hline & EES mass $(\mathrm{kg})$ & 211 & 222 & 333 & 422 \\
\hline & Cost (\$/day) & 4.80 & 4.81 & 5.45 & 9.26 \\
\hline & MPGe & 139 & 138 & 132 & 127 \\
\hline \multirow{6}{*}{ HEES } & $Q_{B}(\mathrm{Ah})$ & 92 & 92 & 92 & 185 \\
\hline & $Q_{S C}(\mathrm{Ah})$ & 0.35 & 0.47 & 0.52 & 0.60 \\
\hline & EES volume (L) & 139 & 147 & 151 & 272 \\
\hline & EES mass (kg) & 192 & 200 & 203 & 380 \\
\hline & Cost (\$/day) & 3.95 & 3.99 & 4.01 & 7.18 \\
\hline & MPGe & 177 & 177 & 177 & 167 \\
\hline \multirow{2}{*}{$\begin{array}{c}\text { ICE } \\
\text { vehicle }\end{array}$} & Gas mileage (MPG) & $31^{1}$ & $26^{2}$ & $16^{3}$ & $16^{3}$ \\
\hline & Gas cost (\$/day) & 3.73 & 4.44 & 7.22 & 7.22 \\
\hline
\end{tabular}


(a) Normalized cost per cycle

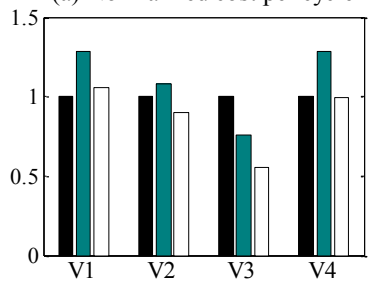

(b) Normalized MPG/MPGe

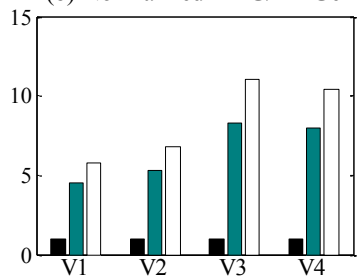

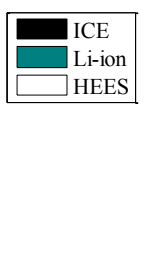

(c) System Mass (kg)

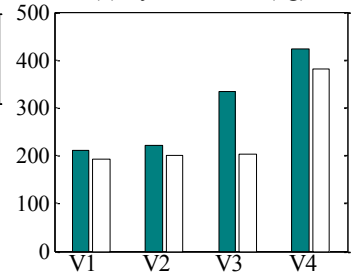

(d) System Volume (L)

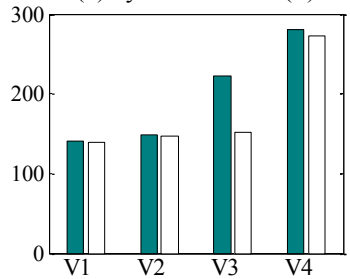

Figure 4. Simulation Results.

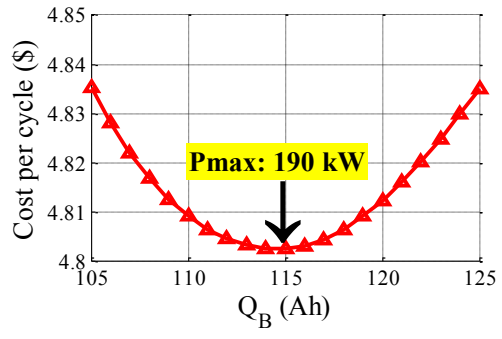

Figure 5. The optimal cost per day as a function of battery capacity in Li-ion battery-only system.

the range constraint since $\mathrm{V} 1, \mathrm{~V} 2$, and $\mathrm{V} 3$ have similar sizing.

2) Comparing the results of V1, V2, and V3 shown in Table 7 , we find that with the increase of battery bank capacity, the homogeneous EES system does not have MPGe gain mainly because of the system mass increase. On the contrary, the proposed HEES system is able to alleviate the MPGe decrease caused by additional system mass. The main reasons are the extension of Li-ion battery lifetime and the improvement of discharge/charge efficiency.

Figure 5 shows the overall amortized cost per $\mathrm{d} / \mathrm{c}$ cycle for a Liion battery-only EES as a function of the battery capacity, which satisfies the range requirement. An optimized 100-mile range battery-only EV (V1 in Table 7) with the minimum cost of $\$ 4.8$ per $\mathrm{d} / \mathrm{c}$ cycle has a maximum power output of $190 \mathrm{~kW}$. This battery-only EV is optimal if we only consider the range and ignore the power constraint. The EV HEES systems for V2 and V3 with the same range both outperform the battery-only optimal model with not only less cost per cycle but also higher maximum output power. This means the higher cost of battery-only systems is not mainly due to the maximum power requirement, but its lower efficiency. Therefore, we have the third conclusion as follows:

3) The proposed EV HEES system is able to achieve not only lower cost but higher power output compared to a Li-ion battery-only EES system.

\subsection{Comparison with ICE Vehicles}

Conventional ICE vehicles have an operational cost highly dependent on the gasoline price. Based on current national average gas and electricity price as listed in Table 5 , the EV models with designed HEES system achieve more than eight times fuel economy on average compared to conventional cars with equal maximum power output, as shown in Table 7 and Figure 4(b). We calculate the amount of gas consumption based on the city/highway combination gas mileage values of a vehicle with similar maximum power output ${ }^{1}$.

\footnotetext{
${ }^{1}$ Since it is difficult to find a commercial ICE vehicle with exactly the same maximum power output and vehicle mass, we only match the maximum power output in selecting the corresponding ICE vehicles.
}

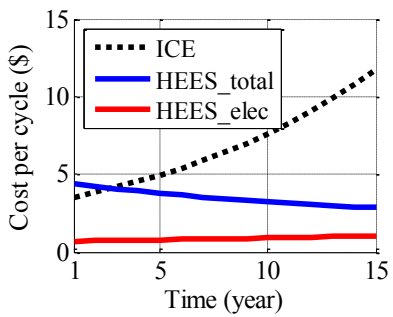

Figure 7. Future cost of ICE and HEES systems. igure 6. Cost decomposition of EV HEES system.
The amortized overall cost per $\mathrm{d} / \mathrm{c}$ cycle of EVs does not always have an advantage over ICE-powered vehicles (Figure 4(a)). The main reason is the high cost of the EES elements. The capital cost of the HEES system accounts for around $80 \%$ of the total cost as shown in Figure 6. However, the gasoline price has been increasing by $9 \%$ each year for the past ten years, while Li-ion battery and supercapacitor unit price is decreasing. Although the electricity unit price is also rising by roughly $3 \%$ each year, the operational cost takes only a small fraction of the overall amortized cost, as shown in Figure 6.

Take the EV model V1 for an example, in which the HEES has $5.9 \%$ more cost per day than an ICE vehicle with equal power output based on current gas/electricity price. Assume 9\% and 3\% for gas and electricity price annual growth rate, respectively, and $5 \%$ annual decrease for the unit prices of batteries and supercapacitors. Figure 7 shows the projected cost per day estimation. From the third year on, an EV with the proposed HEES system is able to outperform the ICE vehicles in overall cost per day. In conclusion, the EV HEES has the potential of making higher profits in the future.

\subsection{Impact of Discrete Sizing}

In practice, sizing the battery and supercapacitor pack is a discrete problem rather than a continuous problem. The proposed design approach might end up in a result that needs to be rounded up to the nearest integer number of battery/supercapacitor cells.

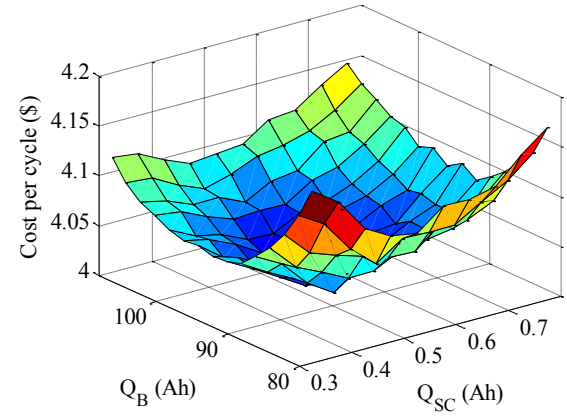

Figure 8. Cost per $\mathrm{d} / \mathrm{c}$ cycle as a function of battery capacity and supercapacitor capacity. 
We show that this approximation leads to negligible error in cost estimation. Figure 8 shows the estimated daily cost of an EV with specification V3 (100-mile AER, $250 \mathrm{~L}$ volume, and $300 \mathrm{~kW}$ maximum power) with different battery bank and supercapacitor bank capacities. The surface clearly indicates that the cost is in general smooth without any anomalies, and unimodal with regard to battery capacity and supercapacitor capacity. A $6.5 \%$ difference in both bank's capacities merely results in less than $1.4 \%$ increase in the estimated daily cost. Therefore, discrete sizing of the storage banks does not impose any additional difficulties to our proposed approach.

\section{CONCLUSIONS}

Designing a cost-efficient and high-performance energy storage system for electric vehicles (EVs) has emerged as a critical problem in the EV technology development. As recent work points to the benefits of hybrid electrical energy storage (HEES) systems, this paper formulates an optimal design problem of a costefficient HEES system for EVs equipped with a Li-ion battery bank and a supercapacitor bank. We formulate the problem of minimizing the total system cost, which is the sum of the capital cost and the operational cost, and introduce a combined gradient descent and simulated annealing algorithm to derive the optimal system design. Simulation results with real vehicle models and parameters show that the proposed EV HEES system outperforms a homogeneous Li-ion battery-only system by $20.91 \%$ less overall cost and $30.29 \%$ higher fuel economy on average.

\section{REFERENCES}

[1] Affanni, A., Bellini, A., Franceschini, G., Guglielmi, P., \& Tassoni, C. "Battery choice and management for newgeneration electric vehicles." Industrial Electronics, IEEE Transactions on 52.5 (2005).

[2] Allegre, A. L., Bouscayrol, A., \& Trigui, R. "Influence of control strategies on battery/supercapacitor hybrid Energy Storage Systems for traction applications." Vehicle Power and Propulsion Conference, 2009.

[3] Chen, M., \& Rincon-Mora, G. A. "Accurate electrical battery model capable of predicting runtime and IV performance." Energy conversion, IEEE Transactions on 21.2 (2006).

[4] http://www.epa.gov/nvfel/testing/dynamometer.htm\#area.

[5] Eddahech, Akram, et al. "Behavior and state-of-health monitoring of Li-ion batteries using impedance spectroscopy and recurrent neural networks."International Journal of Electrical Power \& Energy Systems 42.1 (2012).

[6] EPA Test Procedures for Electric Vehicles and Plug-in Hybrids, 2011.

[7] Gerssen-Gondelach, S. J., Faaij, A., "Performance of batteries for electric vehicles on short and longer term", Journal of Power Sources 2012.

[8] International Energy Agency, "Technology Roadmap, Electric and Plug-in Hybrid Electric Vehicles", 2009.

[9] Linden, D. \& Reddy, T. B. Handbook of Batteries. McGrewHill Professional, 2001

[10] Lukic, S. M., Cao, J., Bansal, R. C., Rodriguez, F., \& Emadi, A. "Energy storage systems for automotive applications." Industrial electronics, IEEE Transactions on, 55.6 (2008).
[11] Lukic, S. M., Wirasingha, S. G., Rodriguez, F., Cao, J., \& Emadi, A. "Power management of an ultracapacitor/battery hybrid energy storage system in an HEV." Vehicle Power and Propulsion Conference, 2006.

[12] Luo, Z., et al. "Economic analyses of plug-in electric vehicle battery providing ancillary services." Electric Vehicle Conference (IEVC), 2012.

[13] Markel, T., et al. "ADVISOR: a systems analysis tool for advanced vehicle modeling." Journal of power sources 110.2 (2002).

[14] Miller, J. M., Bohn, T., Dougherty, T. J., \& Deshpande, U. "Why hybridization of energy storage is essential for future hybrid, plug-in and battery electric vehicles." Energy Conversion Congress and Exposition, 2009.

[15] Miller, J. M., Deshpande, U., Dougherty, T. J., \& Bohn, T. "Power electronic enabled active hybrid energy storage system and its economic viability." Applied Power Electronics Conference and Exposition, 2009.

[16] Ortúzar, M., Moreno, J., \& Dixon, J. "Ultracapacitor-based auxiliary energy system for an electric vehicle: Implementation and evaluation." Industrial Electronics, IEEE Transactions on 54.4 (2007).

[17] Park, S., Kim, Y., \& Chang, N. "Hybrid energy storage systems and battery management for electric vehicles." Proceedings of the 50th Annual Design Automation Conference. ACM, 2013.

[18] Pedram, M., Chang, N., Kim, Y., \& Wang, Y. "Hybrid electrical energy storage systems." Low-Power Electronics and Design (ISLPED), 2010 ACM/IEEE International Symposium on.

[19] Shin, D., et al. "Battery-supercapacitor hybrid system for high-rate pulsed load applications." Design, Automation \& Test in Europe Conference \& Exhibition (DATE), 2011.

[20] Wang, L., Collins, E. G., \& Li, H. "Optimal design and realtime control for energy management in electric vehicles." Vehicular Technology, IEEE Transactions on 60.4 (2011).

[21] Wong, J. Y., Theory of ground vehicles. Wiley-Interscience, 2001

[22] Xie, Q., et al. "State of health aware charge management in hybrid electrical energy storage systems." Proceedings of the Conference on Design, Automation and Test in Europe. EDA Consortium, 2012.

[23] Zhang, Y., \& Wang, C. Y. "Cycle-life characterization of automotive lithium-ion batteries with $\mathrm{LiNiO} 2$ cathode." Journal of the Electrochemical Society 156.7 (2009).

[24] Zhu, D., et al. (2013). Maximizing return on investment of a grid-connected hybrid electrical energy storage system. In $A S P-D A C$ (pp. 638-643).

[25] Zhu, D., et al. (2013, September). Designing a residential hybrid electrical energy storage system based on the energy buffering strategy. In Proceedings of the Ninth IEEE/ACM/IFIP International Conference on Hardware/Software Codesign and System Synthesis (p. 32). IEEE Press. 